\title{
The burden of generalized anxiety disorder in Canada
}

\section{Louise Pelletier, MD (1); Siobhan O'Donnell, MSc (1); Louise McRae, BSc (1); Jean Grenier, PhD (2,3)}

This article has been peer reviewed.

\begin{abstract}
Introduction: Although generalized anxiety disorder (GAD) is common and disabling, there are few Canadian studies on this mental illness. We compared the characteristics, health status, health services use and health care needs of Canadians with GAD to those with depression.
\end{abstract}

Methods: Data are from the 2012 Canadian Community Health Survey-Mental Health, which surveyed a nationally representative sample of Canadians aged 15 years and older ( $n=23$ 709; response rate of $68.9 \%$ ). The respondents we studied had selfreported symptoms compatible with GAD and/or major depressive episode (MDE) in the preceding 12 months ( $\mathrm{n}=1598)$. Estimates were weighted to represent the Canadian household population. We performed descriptive and multinomial multivariate logistic regression analyses.

Results: In 2012, an estimated 700000 (2.5\%) Canadians aged 15 years and older reported symptoms compatible with GAD in the previous 12 months. MDE symptoms co-occurred in $50 \%$ of these individuals. Those with GAD only reported fair/poor perceived health $(29.7 \%)$, moderate to severe psychological distress $(81.2 \%)$ and moderate to severe disability $(28.1 \%)$ comparable to (or even slightly worse) than those with MDE only $(24.7 \%, 78.8 \%$ and $24.8 \%$ respectively). Those with comorbid GAD and MDE demonstrated the worst health outcomes; $47.3 \%$ of them reported fair/poor perceived health, $94.0 \%$ reported moderate to severe psychological distress and $52.4 \%$ reported moderate to severe disability.

Nearly $50 \%$ of those with comorbid GAD and MDE reported that their need for health care was not met or only partially met, compared to about $30 \%$ of those with GAD or MDE only.

Conclusion: While GAD is associated with levels of distress and disability comparable to (or slightly worse) than those affected by MDE only, the health status of those with comorbid disease is significantly worse than those with GAD or MDE only. Improved diagnosis, screening for comorbidity and management are essential to minimize the impacts of this mental illness.

Keywords: generalized anxiety disorder, impact, prevalence, disability, Canada, major depressive episode

\section{Introduction}

In 2012, an estimated 2.4 million (or $8.7 \%$ ) Canadians aged 15 years and older reported symptoms compatible with generalized anxiety disorder (GAD) during their lifetime. Among these individuals, $30 \%$ (or $2.6 \%$ of Canadians) reported symptoms in the 12 months preceding the survey. ${ }^{1}$ This was the very first survey to provide national population estimates for GAD in Canada. Epidemiological studies using comparable methodology conducted in the United States, Europe and Australia found similar 12-month prevalence estimates $(1 \%-4 \%)$. $^{2,3,4}$

\section{Highlights}

- In 2012, an estimated 700000 (2.5\%) Canadians aged 15 years and older reported symptoms compatible with generalized anxiety disorder (GAD) in the previous 12 months, of whom $50 \%$ reported co-occurring symptoms compatible with a major depressive episode (MDE).

- While individuals with GAD only reported levels of fair/poor mental health, psychological distress and disability comparable to those affected by MDE only, those with comorbid GAD and MDE demonstrated significantly worse overall health outcomes.

- Nearly $50 \%$ of those with comorbid GAD and MDE reported that their need for mental health care was either not met or only partially met, compared to about $30 \%$ of those with GAD only or MDE only.

From a clinical perspective, individuals with GAD experience excessive anxiety and worry about a variety of topics (i.e. school, work and relationships), life events or daily activities. Worry occurs more days than not, for at least six months, and is clearly excessive and difficult to control. ${ }^{5,6}$ In order to meet the diagnostic criteria for GAD, symptoms of excessive anxiety and worry must be associated with at least three of the following six symptoms in adults and at least one in children: restlessness, fatigue, trouble concentrating, irritability, muscle tension or sleep problems. The anxiety, worry or physical symptoms cause clinically significant distress or impairment in important areas of daily functioning, and the disturbance is not attributable to the physiological effects of a substance or another medical condition. ${ }^{6}$

\footnotetext{
Author references:

1. Centre for Chronic Disease Prevention, Public Health Agency of Canada, Ottawa, Ontario, Canada

2. Institut de recherche de l'Hôpital Montfort (IRHM), C.T. Lamont Primary Health Care Research Centre, Ottawa, Ontario, Canada

3. Department of Family Medicine, University of Ottawa, Ottawa, Ontario, Canada
}

Correspondence: Louise Pelletier, Centre for Chronic Disease Prevention, Public Health Agency of Canada, 785 Carling Avenue, AL: 6806A, Ottawa, ON K1A 0K9; Tel: 613-960-5339; Email: louise.pelletier@phac-aspc.gc.ca 
GAD usually begins in late adolescence or in the twenties; however, it can develop at any time of life. ${ }^{7}$ A number of adults with GAD indicate that they have been worriers almost their entire life. Because excessive anxiety and worry is not clearly defined and is usually insidious in nature among those with early onset, it usually takes more than 10 years before a person is diagnosed. ${ }^{7}$ On the other hand, those with a later onset, often related to a stressful life event, are more likely to consult a health professional within the first year of symptoms. ${ }^{7}$ GAD has long been considered a chronic condition with waxing and waning of symptoms. However, a few recent longitudinal cohort studies showed that up to $50 \%$ of people with GAD could be symptom-free for extended periods of time, although a number of these individuals will relapse. ${ }^{8,9,10}$ Higher levels of disability are more often associated with an earlier age of symptom onset and a longer duration of untreated symptoms. ${ }^{10,11}$ In addition, the presence of comorbidities is associated with worse health outcomes. ${ }^{6,8}$

One of the main challenges in the early identification of GAD is that affected individuals rarely consult a health professional explicitly for excessive anxiety or worry. ${ }^{12}$ They may instead consult for somatic symptoms such as fatigue, trouble sleeping, headaches, gastrointestinal symptoms or symptoms related to comorbidities. ${ }^{5}$

If symptoms are mild, a person with GAD may develop coping mechanisms, in which case their disorder will likely cause little interference with their daily functioning. In severe cases, however, a person with GAD may become seriously functionally impaired. ${ }^{7}$

GAD frequently co-occurs with other mental health disorders, thus making the diagnosis and treatment more challenging. Some studies have suggested that up to $90 \%$ of individuals with GAD present with comorbid mental disorders during their lifetime, including depression and other anxiety disorders.,12 GAD symptoms can also co-occur with chronic physical health problems such as chronic pain, diabetes and heart disease, and may exacerbate these physical illnesses or interfere with a person's ability to manage them. ${ }^{13}$

Until the last decade, there were doubts that GAD was an entity by itself because it so often presented to health professionals with other mental health disorders, particularly depression. However, a number of studies have since refuted this belief. ${ }^{7,14}$ These studies have also found that the level of impairment among those with pure GAD was equivalent to that of pure major depression or other severely impairing physical diseases and conditions or mental disorders. ${ }^{7,15,16}$ One of the key features of GAD is that while affected individuals rarely seek help for anxiety symptoms, they consume health care resources at a high rate, and account for a disproportionally high number of health care visits. ${ }^{4,16,17}$

Although depression has been widely studied, there is a paucity of epidemiological studies on GAD in Canada. Using data from a sample designed to be nationally representative of Canadians aged 15 years and older, our objectives in this study were (1) to compare the sociodemographic, behavioural and health characteristics of individuals with symptoms compatible with GAD (with or without comorbid MDE) in the preceding 12 months to those with MDE only; (2) to compare the health status, health services use and perceived need for health care among individuals with GAD (with or without comorbid MDE) to those with MDE only; and (3) to determine whether age differences exist in the associations between health status or health services use and these mental disorders.

\section{Methods}

\section{Data source and study sample}

The 2012 Canadian Community Health Survey-Mental Health (CCHS-MH) is a cross-sectional survey with a multistage stratified cluster sampling design covering the Canadian population aged 15 years and older living in the 10 provinces. $^{18}$ Exclusions include people living on reserves and Crown lands, homeless people, fulltime members of the Canadian Forces and the institutionalized population, which together represent about $3 \%$ of the target population.

The purpose of the CCHS-MH was to collect information about mental health status, access to and perceived need for formal and informal mental health services and supports, overall functioning and disability, health determinants and sociodemographics.
The overall response rate for the CCHS-MH was $68.9 \%$. For this study, we used the share file ( $\mathrm{n}=23$ 709) and excluded those respondents $(n=293)$ with missing responses to either GAD or MDE symptombased measures, which resulted in a total study sample of 23416 .

More detailed information on the CCHS-MH, including the questionnaire, may be found at http://www23.statcan.gc.ca/imdb/p2SV .pl? Function $=$ getSurvey $\&$ SDDS $=5015$ \&Item_Id = 119790\&lang = en

\section{GAD and MDE symptom-based measures}

The CCHS-MH/World Health Organization Composite International Diagnostic Interview (WHO-CIDI) criteria are a modified version of the WHO-CIDI. The WHO-CIDI is a standardized instrument for the assessment of mental disorders and conditions according to an operationalization of the definitions and criteria of the Diagnostic and Statistical Manual of Mental Disorders, Fourth Edition (DSM-IV). ${ }^{19,20}$ It is designed to measure the prevalence of mental disorders at the community level, and can be administered by lay interviewers.

We considered respondents to have symptoms compatible with GAD if they met the CCHS-MH/WHO-CIDI criteria for GAD in the preceding 12 months. ${ }^{18}$ Similar procedures were used to identify those with symptoms compatible with MDE in the preceding 12 months. See Box 1 for more information.

\section{Sociodemographics}

The sociodemographic characteristics we studied included sex (women, men); age (15-29, 30-49, 50 + years and mean age); marital status (single, divorced/separated/ widowed, married/common law); respondent's highest level of education (less than secondary, secondary graduate, some post-secondary, post-secondary graduate); adjusted household income quintiles; employment status in the previous week (student, did not work including permanently disabled and elderly, worked); immigrant status (yes, no); Aboriginal status (yes, no); and geography (urban, rural).

To compute the adjusted household income quintiles, we divided respondents into income quintiles based on the adjusted ratio of their total household income to the low income cut-off corresponding to their household and community size, as derived by Statistics Canada. ${ }^{21}$ 


\section{BOX 1}

\section{CCHS-MH/WHO-CIDI criteria for GAD, MDE and substance use disorder ${ }^{21}$}

The 2012 Canadian Community Health Survey-Mental Health (CCHS-MH) used a modified version of the World Health Organization - Composite International Diagnostic Interview 3.0 (WHO-CIDI) to classify people with select mental or substance use disorders. Although this is not a clinical diagnosis, this is a standardized instrument that is typically used to assess mental disorders in population surveys according to the Diagnostic and Statistical Manual of Mental Disorders, Fourth Edition (DSM-IV) criteria.

\section{Generalized anxiety disorder (GAD)}

For the purposes of this survey, respondents who experienced the following CCHS-MH/WHO-CIDI lifetime criteria associated with generalized anxiety disorder reported

- excessive anxiety and worry and anxiety about at least one event or activity that lasted at least six months;

- finding it difficult to control the worry;

- the anxiety and the worry were associated with three or more of the symptoms associated with anxiety; and

- the anxiety, worry, or physical symptoms caused clinically significant distress or significant impairment in social, occupational, or other important areas of functioning.

For the purposes of this survey, respondents who experienced the following CCHS-MH/WHO-CIDI 12-month criteria associated with generalized anxiety disorder reported

- meeting the criteria for lifetime generalized anxiety disorder;

- having an episode of generalized anxiety lasting at least six months in the 12 months before the interview; and

- clinically significant distress or impairment in social, occupational or other important areas of functioning.

\section{Major depressive episode (MDE)}

For the purposes of this survey, respondents who experienced the following CCHS-MH/WHO-CIDI lifetime criteria associated with major depressive episode, reported

- a period of two weeks or more with depressed mood or loss of interest or pleasure AND at least five additional symptoms;

- clinically significant distress or social or occupational impairment; and

- the symptoms are not better accounted for by bereavement.

For the purposes of this survey, respondents who experienced the following CCHS-MH/WHO-CIDI 12-month criteria associated with major depressive episode

- met the criteria for lifetime diagnosis of major depressive episode (see above);

- reported an episode in the past 12 months; and

- reported marked impairment in occupational or social functioning.

\section{Any substance use disorder (either dependence or abuse) in the past 12 months}

"Any substance use disorder (either dependence or abuse) in past 12 months" refers to the use of any of the following: alcohol, cannabis, cocaine, club drugs, hallucinogens, heroin or opium, inhalant or solvent use, or nonmedical use of stimulants or analgesics. Respondents were defined as having symptoms compatible with a substance use disorder if they met the CCHS-MH/WHO-CIDI criteria for abuse or dependence of alcohol or drugs in the past 12 months.

- Dependence is characterized by a recurrent pattern of use where at least three of the following occur in the same 12-month period: increased tolerance, withdrawal, increased consumption, unsuccessful attempts to quit, a lot of time lost recovering or using, reduced activities and continued use despite persistent physical or psychological problems caused or intensified by substance use.

- Abuse is characterized by a recurrent pattern of use where at least one of the following occurs: failure to fulfill major roles at work, school or home; use in physically hazardous situations; recurrent alcohol related problems; or continued use despite social or interpersonal problems caused or intensified by alcohol. By definition, respondents who meet the criteria for substance dependence are excluded from meeting the criteria for substance abuse. 


\section{Behavioural and health characteristics}

Smoking status was defined as "daily," "occasional/former" or "never." The number of physical comorbidities was based on self-reported, health-professional diagnosis of asthma, chronic obstructive pulmonary disease, arthritis, back problems, Crohn's disease, ulcerative colitis, diabetes, epilepsy, heart disease, cancer, stroke, Alzheimer's disease or any other dementia. Each disease was counted as a single physical comorbidity and the following three categories were reported: "none," " 1 or 2 " and " 3 or more."

Respondents were considered to have symptoms compatible with a substance use disorder if they met the CCHS-MH/ WHO-CIDI criteria for abuse of or dependence on alcohol or drugs in the past 12 months (Box 1).

\section{Health status}

Perceived health is an indicator of overall health status. Perceived health was measured by asking respondents "In general, would you say your health is excellent, very good, good, fair, or poor?" Suboptimal health status was defined as having "fair" or "poor" health.

Self-rated mental health was measured by asking respondents "In general, would you say your mental health is excellent, very good, good, fair, or poor?" Suboptimal mental health was defined as having "fair" or "poor" mental health.

"Level of psychological distress in the past month" was determined using the Kessler 6 (K6) instrument, which asks questions about feeling "nervous, hopeless, restless or fidgety, so depressed that nothing can cheer you up, everything was an effort and/or worthless" in the past month. ${ }^{22}$ Scores range from 0 to 24 and are categorized as "no distress," "moderate" and "severe mental distress." 23

"Level of disability in the last 30 days" was determined using the WHO Disability Assessment Schedule 2.0, which includes six domains of disability: cognition, mobility, self-care, getting along, life activities and participation. The overall score ranges from 0 (no disability) to 100 (full disability). Scoring is based on the recommended method outlined in the WHODAS 2.0 manual. ${ }^{24}$ The categories were "no disability," "mild disability," "moderate disability" and "severe/extreme disability."25
Health professional consults, and perceived need for mental health care

Health professional consults for mental health issues in the past 12 months were determined by asking respondents if they had seen or talked on the telephone to any of the following people in the past 12 months about problems with their emotions, mental health or use of alcohol or drugs: psychiatrist; psychologist; family doctor or general practitioner; nurse; or social worker, counsellor or psychotherapist. Individuals were considered to have consulted a mental health professional in the past 12 months if they responded "yes" to the above question for psychiatrist, psychologist, social worker, counsellor or psychotherapist.

Overall perceived need for mental health care in the past 12 months was determined by grouping respondents into categories based on whether a need was reported (i.e. for information, medication, counselling or other), and if so, whether their needs were met, partially met or unmet.

\section{Statistical analysis}

To account for sample allocation and survey design, all estimates were weighted using survey weights generated by Statistics Canada to represent the Canadian household population aged 15 years or older in the 10 provinces in $2012 .{ }^{18}$ Furthermore, variance estimates $(95 \%$ CIs and coefficients of variation) were generated through bootstrap weights provided with the data. ${ }^{26}$

We performed cross-tabulation descriptive analyses to describe respondents reporting symptoms compatible with GAD only, comorbid GAD and MDE, and MDE only in the 12 months preceding the survey. Rao-Scott chi-square goodness-of-fit test and linear regression analysis were used to explore the relationship between categorical and continuous (i.e. mean age) respondent characteristics, respectively, and the aforementioned subgroups. Significance was defined as a $p$-value of $<.01$.

We used multinomial multivariate logistic regression analysis to compare the health status, health professional consults and perceived need for mental health care among those reporting symptoms compatible with GAD only, comorbid GAD and MDE versus MDE only in the past 12 months.
Selected covariates were based on the literature and included sex, age, marital status, education level, household income quintiles, employment status, immigrant and Aboriginal statuses, smoking, alcohol and substance use disorder and physical comorbidities. $^{27,28}$

Finally, we tested for interactions between age and the different health status and service use outcomes, adjusting for sociodemographic and health characteristic variables, for the specified mental disorders (i.e. GAD only, comorbid GAD and MDE, and MDE only). Significant models were determined by $p$-value $<.01$.

We performed the analyses with SAS Enterprise Guide version 5.1 (SAS Institute, Cary, NC, USA).

\section{Results}

Prevalence, sociodemographic, behavioural and health characteristics

Among Canadians aged 15 years and older, the prevalence of symptoms compatible with GAD in the preceding 12 months was $2.5 \%$; about half of these individuals also reported symptoms compatible with MDE (Table 1).

The relationship between the subgroups of interest (GAD only, comorbid GAD and MDE, and MDE only) and the sociodemographic, behavioural and health characteristics studied were statistically significant for age and number of physical comorbidities only (Table 1).

Compared to those with MDE only, those with GAD only were older (mean age of 43.8 vs. 38.0 years), and more likely to have at least one physical comorbidity $(65.9 \%$ vs. $49.0 \%)$. The latter finding is likely due to the confounding effect of age. Similarly, compared to those with MDE only, those with comorbid GAD and MDE were also slightly older (42.2 years) and more likely to have at least one physical comorbidity $(68.4 \%)$.

\section{Health status, health professional consults and perceived need for mental health care}

Based on results from the subgroup analyses (GAD only, GAD and MDE, and MDE only), those with GAD only reported levels of fair/poor perceived health (29.7\%), moderate to severe psychological distress $(81.2 \%)$ and moderate to severe disability 
TABLE 1

Sociodemographic and health characteristics of those with symptoms compatible with GAD only, comorbid GAD and MDE, and MDE only, household population aged 15 years and older, Canada excluding the territories, 2012

\begin{tabular}{|c|c|c|c|c|}
\hline \multirow{2}{*}{$\begin{array}{l}\text { Sociodemographic and } \\
\text { health characteristics }\end{array}$} & $\begin{array}{c}\text { GAD only } \\
(n=346 ; 1.2 \%)\end{array}$ & $\begin{array}{l}\text { GAD and MDE } \\
(\mathrm{n}=358 ; 1.3 \%)\end{array}$ & $\begin{array}{c}\text { MDE only } \\
(n=894 ; 3.4 \%)\end{array}$ & \multirow{2}{*}{$p$-value } \\
\hline & $\%(95 \% \mathrm{Cl})$ & $\%(95 \% \mathrm{Cl})$ & $\%(95 \% \mathrm{CI})$ & \\
\hline \multicolumn{5}{|l|}{ Sex } \\
\hline Women & $60.2(52.6-67.7)$ & $68.2(60.7-75.7)$ & $61.2(56.2-66.3)$ & .23 \\
\hline \multicolumn{5}{|l|}{ Age } \\
\hline $\begin{array}{l}\text { Mean age (years) } \\
\text { Age groups (years) }\end{array}$ & $43.8(41.2-46.5)$ & $42.2(40.0-44.4)$ & $38.0(36.5-39.5)$ & $<.001$ \\
\hline $15-29$ & $22.8(17.2-28.4)$ & $22.7(15.9-29.5)$ & $37.4(32.2-42.6)$ & \multirow[t]{3}{*}{$<.001$} \\
\hline $30-49$ & $37.9(30.8-45.1)$ & $48.6(40.5-56.6)$ & $39.6(34.1-45.2)$ & \\
\hline $50+$ & $39.3(31.6-47.0)$ & $28.8(22.3-35.3)$ & $23.0(19.3-26.7)$ & \\
\hline \multicolumn{5}{|l|}{ Marital status } \\
\hline Single & $30.8(23.9-37.7)$ & $32.4(25.3-39.4)$ & $43.4(38.1-48.6)$ & \multirow[t]{3}{*}{.05} \\
\hline $\begin{array}{l}\text { Divorced/separated/ } \\
\text { widowed }\end{array}$ & $20.8(14.0-27.6)$ & $19.7(14.1-25.4)$ & $16.6(11.9-21.2)$ & \\
\hline Married/common-law & $48.4(40.3-56.6)$ & $47.9(39.7-56.1)$ & $40.1(34.9-45.3)$ & \\
\hline \multicolumn{5}{|l|}{ Education (respondent) } \\
\hline Less than secondary & $15.0^{\mathrm{E}}(9.2-20.9)$ & $18.2(12.9-23.6)$ & $18.2(14.3-22.1)$ & \multirow[t]{4}{*}{.50} \\
\hline Secondary graduate & $15.3^{\mathrm{E}}(9.1-21.4)$ & $14.8^{\mathrm{E}}(9.4-20.3)$ & $16.1(12.6-19.6)$ & \\
\hline Some post-secondary & $6.5^{\mathrm{E}}(3.3-9.7)$ & $11.7^{\mathrm{E}}(5.7-17.8)$ & $11.8(8.3-15.3)$ & \\
\hline Post-secondary graduate & $63.2(55.5-70.9)$ & $55.2(47.6-62.8)$ & $53.9(48.6-59.2)$ & \\
\hline \multicolumn{5}{|l|}{ Income quintile (household) } \\
\hline $1^{\text {st }}$ & $22.8(17.0-28.6)$ & $36.6(28.5-44.6)$ & $32.0(26.8-37.2)$ & \multirow[t]{5}{*}{.07} \\
\hline $2^{\text {nd }}$ & $21.6(15.6-27.7)$ & $25.5(18.6-32.3)$ & $21.9(17.2-26.6)$ & \\
\hline $3^{\text {rd }}$ & $21.3^{\mathrm{E}}(14.0-28.7)$ & $19.3^{\mathrm{E}}(12.8-25.9)$ & $17.0(13.3-20.6)$ & \\
\hline $4^{\text {th }}$ & $18.2^{\mathrm{E}}(11.7-24.8)$ & $10.2^{\mathrm{E}}(6.1-14.3)$ & $17.0(13.2-20.8)$ & \\
\hline $5^{\text {th }}$ & $16.0^{\mathrm{E}}(10.0-21.9)$ & $8.5^{\mathrm{E}}(4.5-12.4)$ & $12.1(9.0-15.2)$ & \\
\hline \multicolumn{5}{|l|}{ Employment status } \\
\hline Student & $11.9^{\mathrm{E}}(7.9-15.9)$ & $10.7^{\mathrm{E}}(6.4-15.0)$ & $19.2(15.3-23.2)$ & \multirow[t]{3}{*}{.03} \\
\hline Did not work & $45.1(37.3-52.8)$ & $50.6(42.1-59.1)$ & $39.7(34.1-45.2)$ & \\
\hline Worked & $43.0(35.6-50.4)$ & $38.7(29.9-47.5)$ & $41.1(36.0-46.2)$ & \\
\hline \multicolumn{5}{|l|}{ Immigrant status } \\
\hline Immigrant & $16.3^{\mathrm{E}}(9.2-23.4)$ & $14.1^{\mathrm{E}}(7.7-20.4)$ & $17.9(13.4-22.4)$ & \multirow[t]{2}{*}{.65} \\
\hline Non-immigrant & $83.7(76.6-90.8)$ & $85.9(79.6-92.3)$ & $82.1(77.6-86.6)$ & \\
\hline \multicolumn{5}{|l|}{ Aboriginal status } \\
\hline $\begin{array}{l}\text { Aboriginal } \\
\text { Non-Aboriginal }\end{array}$ & $\begin{array}{c}5.6^{\mathrm{E}}(2.6-8.6) \\
94.4(91.4-97.4)\end{array}$ & $\begin{array}{c}7.4^{\mathrm{E}}(3.3-11.4) \\
92.6(88.6-96.7)\end{array}$ & $\begin{array}{c}6.2^{\mathrm{E}}(3.2-9.2) \\
93.8(90.8-96.8)\end{array}$ & .80 \\
\hline \multicolumn{5}{|l|}{ Geographic area } \\
\hline Rural & $18.3^{\mathrm{E}}(12.2-24.3)$ & $17.1 \quad(11.6-22.5)$ & $13.3 \quad(10.2-16.4)$ & \multirow[t]{2}{*}{.19} \\
\hline Urban & $81.8(75.8-87.8)$ & $82.9 \quad(77.5-88.4)$ & $86.7 \quad(83.6-89.8)$ & \\
\hline
\end{tabular}

(28.1\%) comparable to (or even slightly worse than) those with MDE only (respectively, $24.7 \%, 78.8 \%$ and $24.8 \%$ ). However, those with comorbid GAD and MDE demonstrated worse health status, irrespective of the measure studied, compared to those affected by MDE only. About half of the comorbid group reported suboptimal perceived health, dissatisfaction with life and severe psychological distress; more than two-thirds reported suboptimal mental health; and nearly one-quarter reported severe or extreme disability. The relationship between the subgroups of interest and all health status measures were statistically significant (Table 2).

Interestingly, less than $60 \%$ of individuals with GAD only or with MDE only consulted a health professional, while nearly $75 \%$ of those with comorbid GAD and MDE indicated having consulted. Similarly, a greater proportion of individuals with comorbid GAD and MDE had consulted a mental health professional (40\%), compared to those with MDE only (about 30\%) and those with GAD only (nearly $25 \%$ ).

When asked whether their need for mental health care was met or not, about $30 \%$ of individuals with GAD only and MDE only reported that their needs were either partially met or not met at all, compared to nearly $50 \%$ of those with comorbid GAD and MDE. The relationships between the subgroups of interest and health professional consults or perceived need for care were all statistically significant (Table 3 ).

Finally, upon controlling for all sociodemographic, behavioural and health characteristics, all health status measures studied were similar (or worse, in the case of moderate disability level) for those with GAD only compared to those with MDE only (Table 4). However, those with comorbid GAD and MDE were about 2.5 times more likely to report fair/poor mental health, 8 times more likely to report severe psychological distress, and nearly 10 times more likely to experience severe levels of disability compared to those with MDE only. Also, they were about 2 times more likely to report their need for care was either partially met or not met at all.

We observed no significant age-related effects (data not shown).

As expected, those with GAD (with or without MDE) demonstrated significantly 
TABLE 1 (continued)

Sociodemographic and health characteristics of those with symptoms compatible with GAD only, comorbid GAD and MDE, and MDE only, household population aged 15 years and older, Canada excluding the territories, 2012

\begin{tabular}{|c|c|c|c|c|}
\hline \multirow{2}{*}{$\begin{array}{l}\text { Sociodemographic and } \\
\text { health characteristics }\end{array}$} & $\begin{array}{c}\text { GAD only } \\
(\mathrm{n}=346 ; 1.2 \%)\end{array}$ & $\begin{array}{l}\text { GAD and MDE } \\
(\mathrm{n}=358 ; 1.3 \%)\end{array}$ & $\begin{array}{c}\text { MDE only } \\
(\mathrm{n}=894 ; 3.4 \%)\end{array}$ & \multirow{2}{*}{$p$-value } \\
\hline & $\%(95 \% \mathrm{Cl})$ & $\%(95 \% \mathrm{Cl})$ & $\%(95 \% \mathrm{Cl})$ & \\
\hline \multicolumn{5}{|l|}{ Smoking status } \\
\hline Daily & $22.0(15.9-28.1)$ & $33.0(25.8-40.1)$ & $26.0(21.5-30.4)$ & \multirow[t]{3}{*}{.04} \\
\hline Occasional/former & $33.7(26.7-40.8)$ & $38.7(31.0-46.4)$ & $40.7 \quad(35.7-45.6)$ & \\
\hline Never & $44.3(36.1-52.4)$ & $28.4(20.6-36.1)$ & $33.4(27.7-39.1)$ & \\
\hline \multicolumn{5}{|l|}{ Substance use disorder } \\
\hline Yes & $10.2^{\mathrm{E}}(6.1-14.3)$ & $17.6^{\mathrm{E}}(11.8-23.3)$ & $16.0(11.7-20.2)$ & \multirow[t]{2}{*}{.12} \\
\hline No & 89.8 (85.7-93.9) & $82.4(76.7-88.2)$ & $84.0 \quad(79.8-88.3)$ & \\
\hline \multicolumn{5}{|l|}{ Physical comorbidities } \\
\hline $3+$ & $18.1^{\mathrm{E}}(11.0-25.3)$ & $19.3(13.1-25.5)$ & $8.6(6.1-11.1)$ & \multirow[t]{3}{*}{$<.001$} \\
\hline $1-2$ & 47.8 (39.9-55.8) & $49.2(41.3-57.1)$ & $40.4 \quad(35.3-45.6)$ & \\
\hline None & $34.1 \quad(26.9-41.2)$ & $31.6(23.5-39.7)$ & $51.0(45.7-56.2)$ & \\
\hline
\end{tabular}

Abbreviations: $\mathrm{Cl}$, confidence interval; GAD, generalized anxiety disorder; MDE, major depressive episode.

Notes: $\mathrm{n}$ are based on unweighted numbers and proportions (\%), means and $95 \% \mathrm{Cls}$ are based on weighted data. This table only presents data of the last 12 months $(n=1598)$.

${ }^{\mathrm{E}} \mathrm{High}$ sampling variability (coefficient of variation between $16.6-33.3 \%$ ).

TABLE 2

Health status factors for those with symptoms compatible with GAD only, comorbid GAD and MDE, and MDE only, household population aged 15 years and older, Canada excluding the territories, 2012

\begin{tabular}{|c|c|c|c|c|}
\hline \multirow{2}{*}{ Health status factors } & $\begin{array}{c}\text { GAD only } \\
(n=346 ; 1.2 \%)\end{array}$ & $\begin{array}{l}\text { GAD and MDE } \\
(n=358 ; 1.3 \%)\end{array}$ & $\begin{array}{c}\text { MDE only } \\
(\mathrm{n}=\mathbf{8 9 4} ; 3.4 \%)\end{array}$ & \multirow[b]{2}{*}{$p$-value } \\
\hline & $\%(95 \% \mathrm{Cl})$ & $\%(95 \% \mathrm{Cl})$ & $\%(95 \% \mathrm{CI})$ & \\
\hline \multicolumn{5}{|l|}{ Perceived health } \\
\hline Fair/poor & $29.7(22.2-37.2)$ & $47.3(39.4-55.1)$ & $24.7(20.2-29.2)$ & $<.001$ \\
\hline \multicolumn{5}{|l|}{ Self-reported mental health } \\
\hline Fair/poor & $44.7(36.8-52.6)$ & $69.9(62.8-77.0)$ & $45.3(40.1-50.5)$ & $<.001$ \\
\hline \multicolumn{5}{|c|}{ Psychological distress in the past month } \\
\hline Severe & $17.8^{\mathrm{E}}(11.4-24.2)$ & $51.1(43.5-58.7)$ & $19.4(15.0-23.7)$ & $<.001$ \\
\hline Moderate & $63.4(55.4-71.4)$ & $42.9(35.1-50.7)$ & $59.4(54.1-64.7)$ & \\
\hline None & $18.8^{\mathrm{E}}(12.3-25.4)$ & $6.0^{\mathrm{E}}(2.9-9.1)$ & $21.2(17.3-25.2)$ & \\
\hline \multicolumn{5}{|l|}{ Disability in the last 30 days } \\
\hline Severe/extreme & NR & $22.2(15.6-28.8)$ & $6.0(4.2-7.9)$ & $<.001$ \\
\hline Moderate & $28.1 \quad(19.5-36.7)$ & 30.2 (22.7-37.6) & $18.8(15.0-22.5)$ & \\
\hline Mild & $47.8 \quad(39.2-56.4)$ & $37.5(29.4-45.5)$ & $43.4(38.0-48.8)$ & \\
\hline None & $19.3(13.2-25.5)$ & $10.2^{\mathrm{E}}(5.5-14.9)$ & $31.8(26.8-36.9)$ & \\
\hline
\end{tabular}

Abbreviations: $\mathrm{Cl}$, confidence interval; GAD, generalized anxiety disorder; MDE, major depressive episode; NR: not reportable (coefficient of variation higher than 33.3).

Notes: $\mathrm{n}$ are based on unweighted numbers and proportions (\%) and $95 \% \mathrm{Cls}$ are based on weighted data.

This table only presents data of the last 12 months $(n=1598)$.

${ }^{\mathrm{E}}$ High sampling variability (coefficient of variation between 16.6-33.3\%). worse perceived health, self-rated mental health, psychological distress and disability levels compared to those with neither GAD nor MDE. Similarly, those with GAD (with or without MDE) were more likely to consult a health professional for their mental health symptoms and their perceived need for mental health care was greater than those without GAD or MDE (data not shown; available upon request from the authors).

\section{Discussion}

In 2012, an estimated 700000 (2.5\%) Canadians aged 15 years and older were affected by symptoms compatible with GAD, with half of these individuals presenting with comorbid MDE symptoms. These estimates may be conservative considering that those affected by GAD (and MDE) have poorer self-reported health and self-reported mental health, and it has been shown that nonresponders have a significantly higher proportion of poor self-rated health even with consideration given to sex, age, country of birth and level of education. ${ }^{29}$ However, to our knowledge, the issue of nonresponse bias among those with different types of mental disorders, such as GAD and MDE, has yet not been studied.

This study highlights the fact that those affected by GAD only reported similar (or even slightly worse) ratings in terms of perceived health, self-reported mental health, psychological distress and disability to those affected by MDE only, as shown elsewhere. ${ }^{3,7,16,30}$

It also demonstrates poorer health-related outcomes among those affected by comorbid GAD and MDE, as well as the considerable limitations in daily life associated with those disorders as evidenced by a high level of disability. These findings support the results of other studies on comorbid anxiety and depression. ${ }^{3,7,12,31-35}$ Therefore, health professionals encountering individuals with either anxiety or depressive symptoms should carefully assess for the presence of comorbid psychological conditions. In addition, considering that nearly $70 \%$ of those with comorbid GAD and MDE had at least one physical chronic condition, and in light of solid evidence elucidating the bidirectional relationship between mental illnesses (specifically depression and anxiety) and physical health outcomes, ${ }^{36}$ particular attention should be given to the prevention 
TABLE 3

Health professional consults and overall perceived need for care for those with symptoms compatible with GAD only, comorbid GAD and MDE, and MDE only, household population aged 15 years and older, Canada excluding the territories, 2012

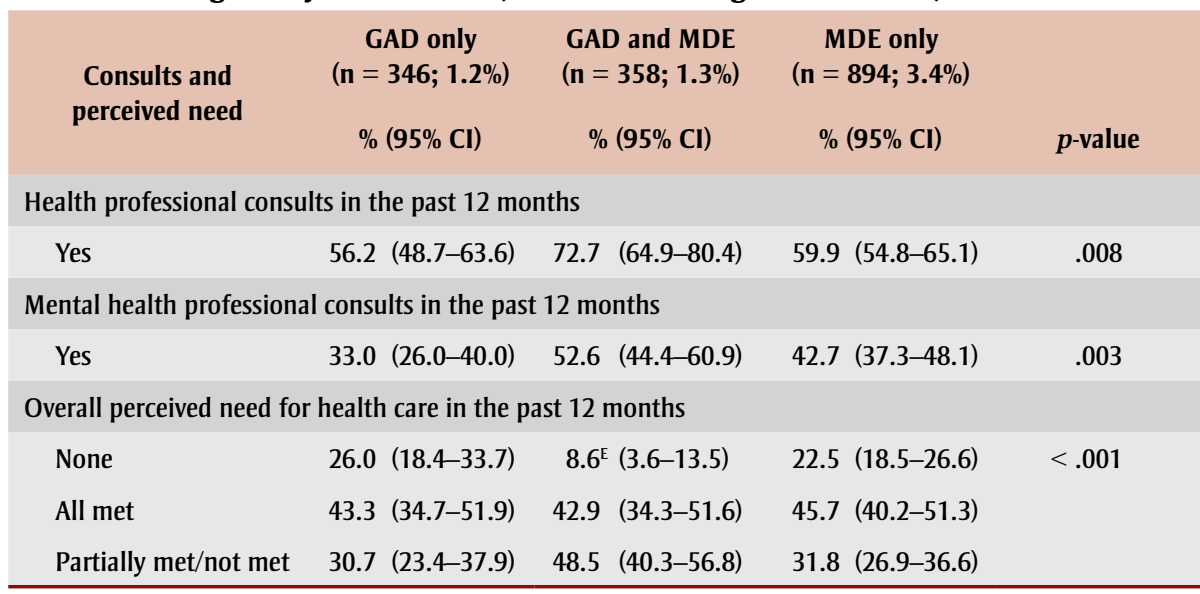

Abbreviations: $\mathrm{Cl}$, confidence interval; GAD, generalized anxiety disorder; MDE, major depressive episode. Notes: $\mathrm{n}$ are based on unweighted numbers and proportions (\%) and $95 \% \mathrm{Cls}$ are based on weighted data. This table only presents data of the last 12 months $(n=1598)$.

${ }^{\mathrm{E}}$ High sampling variability (coefficient of variation between $16.6-33.3 \%$ ).

TABLE 4

Adjusted odds ratio of having symptoms compatible with GAD only or comorbid GAD and MDE versus MDE only, by health status, health professional consults and overall perceived need for care, household population aged 15 years and older, Canada excluding the territories, 2012

\section{OR $(95 \% \mathrm{Cl})$}

\section{Perceived health \\ Fair/poor vs. excellent/very good/good \\ Self-reported mental health \\ Fair/poor vs. excellent/very good/good}

GAD only

GAD and MDE

MDE only

$$
\text { GAD only }
$$

GAD and MDE

MDE only

Psychological distress in the past month

Severe vs. none

GAD only

GAD and MDE

MDE only

Moderate vs. none

GAD only

GAD and MDE

MDE only

GAD only

GAD and MDE

MDE only

$1.2(0.7-2.0)$

$1.9(1.1-3.3)$

Referent

Disability in the last 30 days

Severe/extreme vs. none

GAD only

GAD and MDE

MDE only

GAD only

$1.2(0.8-1.8)$

$2.6(1.6-4.1)$

Referent

Moderate vs. none

Mild vs. none
GAD and MDE

MDE only
$1.8(0.9-3.6)$

7.9 (3.5-17.5)

Referent

$1.8(1.0-3.0)$

$2.4(1.2-5.0)$

Referent

$1.4(0.5-4.1)$

9.8 (4.0-23.9)

Referent

$2.3(1.3-4.3)$

$4.9(2.3-10.4)$

Referent

$1.8(1.0-3.1)$

$2.9(1.4-5.7)$

Referent

Continued on the following page and management of comorbid chronic physical illnesses.

Furthermore, this paper demonstrated that nearly $50 \%$ of those with comorbid GAD and MDE perceived that their need for mental health care was either not met or only partially met compared to about $30 \%$ of those with GAD only or MDE only. While this study did not allow us to estimate the proportion of individuals with GAD being adequately diagnosed and treated, studies have shown that GAD is usually poorly recognized ${ }^{4,16}$ and that up to two-thirds of patients suffering from anxiety disorders do not receive evidencebased treatments..$^{30,33,37}$

\section{Strengths and limitations}

This study has a number of strengths, including the large, population-based sample and the administration of the survey by trained personnel using a structured format.

However, our findings should be interpreted in light of some important limitations. First, the results are based on self-reported data, which is sensitive to social desirability bias, recall bias and conscious nonreporting. Second, the relatively low response rate of $68.9 \%$ is of concern. It could be assumed that those affected by mood and/or anxiety disorders, particularly those with more severe symptoms and poor perceived health, ${ }^{29}$ may be more reticent or ambivalent about participating in such a survey. Consequently, our results may underestimate the true prevalence of these disorders and may be influenced by those who responded, a type of participation bias. While it should be noted that Statistics Canada weighting adjustment strategies help mitigate the impact of overall nonresponse, this may not have had an impact on this particular nonparticipation bias. Third, the disorder type results are based on WHO-CIDI criteria and not on a clinical assessment by a mental health professional. Finally, the results we observed are based on a crosssectional design; therefore, it is not possible to determine whether the associated factors contributed to the development of $\mathrm{GAD}$ and/or MDE or were a consequence of it.

\section{Conclusion}

GAD is common, frequently co-occurs with MDE and can profoundly impact the lives 
TABLE 4 (continued)

Adjusted odds ratio of having symptoms compatible with GAD only or comorbid GAD and MDE versus MDE only, by health status, health professional consults and overall perceived need for care, household population aged 15 years and older, Canada excluding the territories, 2012

\begin{tabular}{|c|c|c|}
\hline & & OR $(95 \% \mathrm{Cl})$ \\
\hline \multicolumn{3}{|c|}{ Health professional consults in the last 12 months } \\
\hline \multirow{3}{*}{ Yes vs. no } & GAD only & $0.8(0.6-1.3)$ \\
\hline & GAD and MDE & $1.6(1.0-2.6)$ \\
\hline & MDE only & Referent \\
\hline \multicolumn{3}{|c|}{ Mental health professional consults in the past 12 months } \\
\hline \multirow{3}{*}{ Yes vs. no } & GAD only & $0.8(0.5-1.2)$ \\
\hline & GAD and MDE & $1.4(0.9-2.3)$ \\
\hline & MDE only & Referent \\
\hline \multicolumn{3}{|c|}{ Overall perceived need for health care } \\
\hline \multirow{3}{*}{ Partially met/not met vs. all met } & GAD only & $1.4(0.8-2.3)$ \\
\hline & GAD and MDE & $1.8(1.1-3.0)$ \\
\hline & MDE only & Referent \\
\hline
\end{tabular}

Abbreviations: $\mathrm{Cl}$, confidence interval; GAD, generalized anxiety disorder; MDE, major depressive episode; OR, odds ratio.

Notes: ORs and 95\% Cls are adjusted for sex, age, marital status, education (respondent), income quintiles (household), employment status, immigrant status, Aboriginal status, geographic region, smoking status, substance use disorder and physical comorbidities, and are based on weighted data.

This table only presents data of the last 12 months $(n=1598)$.

of those affected. While those affected by GAD show similar, or even slightly worse, levels of perceived health, psychological distress and disability to those affected by MDE only, individuals affected by both disorders demonstrated worse health outcomes. Similar to depression, initiatives to improve the recognition and management of GAD and comorbid GAD and MDE are needed to help decrease the severity and persistence of symptoms, and to prevent the onset of secondary mental health disorders or physical chronic diseases.

\section{Acknowledgements}

This research received no specific grant from any funding agency, or commercial or not-for-profit entities.

\section{Conflicts of interest}

None to report.

\section{Authors' contributions}

All authors conceptualised the study, SO analysed the data and LP drafted the paper. All authors contributed to the interpretation of the data and provided comments to the draft paper.

\section{References}

1. Pearson C, Janz T, Ali J. Health at a glance: mental and substance use disorders in Canada. Ottawa $(\mathrm{ON})$ : Statistics Canada; 2013 [Statistics Canada, Catalogue No. 82-624-X].

2. Kessler RC, Chiu WT, Demler O, Walters EE. Prevalence, severity, and comorbidity of 12-month DSM-IV disorders in the National Comorbidity Survey Replication. Arch Gen Psychiatry. 2005;62(6):617-27.

3. Hunt C, Issakidis C, Andrews G. DSM-IV generalized anxiety disorder in the Australian National Survey of Mental Health and Well-Being. Psychol Med. 2002;32(4):649-59.

4. Lieb R, Becker E, Altamura C. The epidemiology of generalized anxiety disorder in Europe. Eur Neuropsychopharmacol. 2005;15(4): 445-52.

5. Hoge EA, Ivkovic A, Fricchione GL. Generalized anxiety disorder: diagnosis and treatment. BMJ. 2012;345: e7500.
6. American Psychiatric Association. Diagnostic and statistical manual of mental disorders. 5th ed. Arlington (VA): American Psychiatric Publishing; 2013.

7. Kessler RC, Keller MB, Wittchen HU. The epidemiology of generalized anxiety disorder. Psychiatr Clin North Am. 2001;24(1):19-39.

8. Bruce SE, Yonkers KA, Otto MW, et al. Influence of psychiatric comorbidity on recovery and recurrence in generalized anxiety disorder, social phobia, and panic disorder: a 12-year prospective study. Am J Psychiatry. 2005;162(6):1179-87.

9. Angst J, Gamma A, Baldwin DS, Ajdacic-Gross V, Rössler W. The generalized anxiety spectrum: prevalence, onset, course and outcome. Eur Arch of Psychiatry Clin Neurosci. 2009;259(1):37-45.

10. Ramsawh HJ, Weisberg RB, Dyck I, Stout R, Keller MB. Age of onset, clinical characteristics, and 15-year course of anxiety disorders in a prospective, longitudinal, observational study. J Affect Disord. 2011;132(1-2):260-4.

11. Kisely S, Scott A, Denney J, Simon G. Duration of untreated symptoms in common mental disorders: association with outcomes: international study. Br J Psychiatry. 2006;189:79-80.

12. Nutt D, Argyropoulos S, Hood S, Potokar J. Generalized anxiety disorder: a comorbid disease. Eur Neuropsychopharmacol. 2006; 16(Suppl 2):S109-S118.

13. Allgulander C. Generalized anxiety disorder: a review of recent findings. J Experiment Clin Med. 2012;4(2): 88-91.

14. Hettema JM. The nosologic relationship between generalized anxiety disorder and major depression. Depress Anxiety. 2008;25(4):300-16

15. Ormel J, Petukhova M, Chatterji S, et al. Disability and treatment of specific mental and physical disorders across the world. Br J Psychiatry. 2008;192(5):368-75.

16. Wittchen, HU. Generalized anxiety disorder: prevalence, burden, and cost to society. Depress Anxiety. 2002; 16(4):162-71. 
17. Bélanger L, Ladouceur R, Morin CM. Generalized anxiety disorder and health care use. Can Fam Physician. 2005;51:1362-3.

18. Statistics Canada. Canadian Community Health Survey (CCHS) - Mental Health Users Guide. Ottawa (ON): Statistics Canada; 2013.

19. Kessler RC, Üstün TB. The World Mental Health (WMH) Survey Initiative Version of the World Health Organization (WHO) Composite International Diagnostic Interview (CIDI). Int J Methods Psychiatr Res. 2004; 13(2):93-121.

20. Kessler RC, Calabrese JR, Farley PA, et al. Composite International Diagnostic Interview screening scales for DSM-IV anxiety and mood disorders. Psychol Med. 2013;43(8):1625-37.

21. Statistics Canada. Canadian Community Health Survey (CCHS) - Mental Health: derived variable (DV) specifications [Internet]. Ottawa (ON): Statistics Canada; 2013 Sep [cited 2015 Oct 22]. Available from: http://odesi1 .scholarsportal.info/documentation /CCHS_syn/2012/CCHS_MH_Derived _Variables.pdf

22. Kessler RC, Barker PR, Colpe LJ, et al. Screening for serious mental illness in the general population Arch Gen Psychiatry. 2003;60(2):184-9.

23. Prochaska JJ, Sung HY, Max W, Shi Y, Ong M. Validity study of the K6 scale as a measure of moderate mental distress based on mental health treatment need and utilization. Int $\mathrm{J}$ Methods Psychiatr Res. 2012;21(2); 88-97.

24. Üstün TB, Kostanjsek, N, Chatterji S, Rehm J. (Eds.). Measuring health and disability: manual for WHO Disability Assessment Schedule (WHODAS 2.0). Geneva (CH): World Health Organization; 2010. Available at: http:// whqlibdoc.who.int/publications /2010/9789241547598_eng.pdf

25. Virués-Ortega J, de Pedro-Cuesta J, Seijo-Martínez M, et al. Prevalence of disability in a composite $\geq 75$-yearold population in Spain: a screening survey based on the International Classification of Functioning. BMC Public Health. 2011;11:176.
26. Rust K, Rao JNK. Variance estimation for complex surveys using replication techniques. Stat Methods Med Res. 1996;5(3):281-310.

27. Mawani FN, Gilmour H. Validation of self-rated mental health. Health Rep. 2010; 21(3):1-15.

28. Toghanian S, Di Bonaventura M, Järbrink K, Locklear JC. Economic and humanistic burden of illness in generalized anxiety disorder: an analysis of patient survey data in Europe. Clinicoecon Outcomes Res. 2014;6(1): 151-63.

29. Lindén-Boström M, Persson C. A selective follow-up study on a public health survey. Eur J Public Health. 2013;23(1):152-7.

30. Hoffman DL, Dukes EM, Wittchen HU. Human and economic burden of generalized anxiety disorder. Depress Anxiety. 2008;25(1):72-90.

31. McEvoy PM, Grove $\mathrm{R}$, Slade $\mathrm{T}$. Epidemiology of anxiety disorders in the Australian general population: findings of the 2007 Australian National Survey of Mental Health and Wellbeing. Aust N Z J Psychiatry. 2011;45(11):957-67.

32. Leray E, Camara A, Drapier D, et al. Prevalence, characteristics and comorbidities of anxiety disorders in France: results from the "Mental Health in General Population” survey (MHGP). Eur Psychiatry. 2011;26(6):339-45.

33. Kroenke K, Spitzer RL, Williams JB, Monahan PO, Löwe B. Anxiety disorders in primary care: prevalence, impairment, comorbidity, and detection. Ann Intern Med. 2007;146(5): 317-25.

34. Belzer K, Schneier FR. Comorbidity of anxiety and depressive disorders: issues in conceptualization, assessment, and treatment. J Psychiatr Pract. 2004;10(5):296-306.

35. Grant BF, Hasin DS, Stinson FS, et al. Prevalence, correlates, co-morbidity, and comparative disability of DSM-IV generalized anxiety disorder in the USA: results from the National Epidemiologic Survey on Alcohol and Related Conditions. Psychol Med. 2005;35(12): 1747-59.
36. Kolappa K, Henderson DC, Kishore SP. No physical health without mental health: lessons unlearned? Bull World Health Organ. 2013;91(1):3-3A.

37. Culpepper, L. Generalized anxiety disorder in primary care: emerging issues in management and treatment. J Clin Psychiatry. 2002;63:35-42. 